\title{
Mutagenesis analysis of T380R mutation in the envelope protein of yellow fever virus
}

\author{
Yan-Jang S Huang ${ }^{1,2}$, John T Nuckols ${ }^{3}$, Kate M Horne ${ }^{2}$, Dana Vanlandingham ${ }^{1,2}$, Mario Lobigs ${ }^{4}$ \\ and Stephen Higgs ${ }^{1,2^{*}}$
}

\begin{abstract}
Background: The RGD motif in the mosquito-borne flaviviruses envelope protein domain III (EDIII) FG loop was shown to bind negatively charged cellular molecules and mediate virus entry in mammals. However, its importance in virus entry in the mosquito has not yet been defined. The sequences of RGD motifs are conserved in JEV-serocomplex members primarily transmitted by Culex mosquitoes but absent from members of the DENV serocomplex, which utilize Aedes mosquitoes as vectors. Interestingly, the RGD sequence is present in the attenuated 17D strain of yellow fever virus as a result of the T380R mutation in the EDIII of Asibi strain following extensive in vitro passage in mice and chicken embryos and was found to contribute to the more rapid clearance in mice challenged with 17D. However, viral infectivity and dissemination in mosquitoes had not been evaluated for this mutant.

Findings: The study utilized the reverse genetics system of YFV and Ae. aegypti RexD WE mosquitoes to assess the impact of a T380R mutation in YFV Asibi and 17D/Asibi M-E chimera. The T380R mutation led to higher infection rates but similar dissemination rates when introduced into the YFV Asibi strain and 17D/Asibi M-E chimera.

Conclusions: While the increase of the positive charge in EDIII may reduce the virulence of YFV in mice, this mutation favored the establishment of the viral infection in Ae. aegypti. However, such gain in viral infectivity did not increase dissemination in infected mosquitoes.
\end{abstract}

Keywords: Yellow fever virus, 17D vaccine, Aedes aegypti

\section{Findings}

The transmission cycles of flaviviruses require successful utilization of cellular receptors in a wide variety of organisms including mammals, avians or arthropods. Although several molecules of mammalian origin have been proposed as putative receptors for mosquitoborne flaviviruses, knowledge about arthropod receptors remains limited [1]. Flavivirus receptor-binding activity in mammals was first shown to be mediated by the domain III of envelope protein (EDIII), eventually localized to the FG loop [2,3]. Although the sequences and structures of the FG loop among mosquito-borne and tick-borne flaviviruses are largely conserved, minor differences were still observed in the FG loop not only

\footnotetext{
* Correspondence: shiggs@bri.ksu.edu

'Biosecurity Research Institute, Kansas State University, Manhattan, KS 66506, USA

${ }^{2}$ Department of Diagnostic Medicine and Pathobiology, College of Veterinary Medicine, Kansas State University, Manhattan, KS 66506, USA

Full list of author information is available at the end of the article
}

between both types of flaviviruses but also among mosquito-borne flaviviruses that utilize different vector species. Interestingly, the FG loops of mosquito-borne flavivirus EDIII are invariably longer than those of tick-borne flavivirus EDIII, which lack the RGD (Arg-Gly-Asp) motif. Inhibition of virus entry by peptides resembling the DENV-2 FG loop in mosquito cells in vitro first suggested its role in determining vector specificity [3]. Alignment of the amino acid sequences of mosquitoborne flaviviruses further demonstrated the RGD motif, especially the Arg and Gly residues, was largely conserved in the mosquito-borne flaviviruses in the JEV serocomplex, which utilize a substantially larger spectrum of host and vector species than other flaviviruses $[4,5]$. However, attenuated YFV 17D strains have the RGD motif while the virulent Asibi parental strain contains a Thr instead of Arg at position 380 [4]. These data highlight the need for understanding the function of the conserved Arg residue, which has been identified as a determinant of binding efficiency to negatively charged host molecules 
Table 1 Ae. aegypti infection rates, calculated as number infected whole mosquitoes and bodies/tested

\begin{tabular}{|c|c|c|c|c|c|c|c|}
\hline \multirow[b]{2}{*}{ Defibrinated sheep blood } & \multirow{2}{*}{$\begin{array}{l}\text { Average blood meal titer }\left(\log \mathrm{TCID}_{50} / \mathrm{ml}\right) \\
\text { N.A. }\end{array}$} & \multicolumn{2}{|l|}{7 d.p.i. } & \multicolumn{2}{|c|}{10 d.p.i. } & \multicolumn{2}{|c|}{14 d.p.i. } \\
\hline & & $0 / 30$ & $(0.0 \%)$ & $0 / 24$ & $(0.0 \%)$ & $0 / 35$ & $(0.0 \%)$ \\
\hline $17 D$ & 6.24 & $9 / 25$ & $(36.0 \%)$ & $8 / 12$ & $(66.7 \%)$ & $8 / 32$ & $(25.0 \%)$ \\
\hline 17D/Asibi M-E & 5.77 & $12 / 29$ & $(41.4 \%)$ & $13 / 24$ & $(54.2 \%)$ & $21 / 39$ & $(53.8 \%)$ \\
\hline 17D/Asibi M-E E T380R & 5.85 & $21 / 23$ & $(91.3 \%)^{\dagger}$ & $10 / 12$ & $(83.3 \%)$ & $14 / 16$ & $(87.5 \%)^{\dagger}$ \\
\hline Asibi & 3.99 & $20 / 33$ & $(60.6 \%)$ & $9 / 18$ & $(50.0 \%)$ & $21 / 38$ & $(52.6 \%)$ \\
\hline Asibi E T380R & 4.21 & $32 / 48$ & $(66.7 \%)$ & $21 / 36$ & $(58.3 \%)$ & $21 / 39$ & $(53.8 \%)$ \\
\hline
\end{tabular}

${ }^{+}$indicates the significant difference by Fisher's exact test from 17D/Asibi M-E chimera.

among flaviviruses [6]. Although characterization of the RGD motif has been performed in JEV, WNV, MVEV and YFV in mice, knowledge of its interaction with arthropod receptors remains limited [6-8]. Length of the corresponding region in DENV-2 EDIII played a more significant role than the sequence in infectivity, and substitution with the sequence of YFV 17D strain did not significantly decrease infectivity in Aedes aegypti. However, the sequence of YFV Asibi strain, which is more infectious and disseminates at higher rates in Ae. aegypti, was not evaluated [9-11]. More importantly, this model provided limited information on the RGD motif function due to the lack of conservation of this motif among the four DENV serotypes. Therefore, we utilized the YFV reverse genetics system to evaluate the impact of the Thr380Arg (T380R) mutation in EDIII on the viral infectivity and dissemination in Ae. aegypti. Such knowledge will increase understanding of the RGD-mo tif-dependent viral entry mechanisms among mosquitoborne flaviviruses utilizing different vectors.

Introduction of T380R mutation in $\mathrm{E}$ of infectious clones of YFV Asibi strain and 17D/Asibi M-E chimera was achieved by subcloning the structural genes into the pGEM ${ }^{\circ}$ T Easy vector (Promega, Madison, WI) followed by PCR site-directed mutagenesis with Quikchange $\mathrm{II}$ XL kit (Stratagene, La Jolla, CA) and ligation of BspEI (New England Biolabs, Ipswich, MA)- and MluI (New England Biolabs)- digested plasmids as previously described [12]. Rescued viruses were obtained via electroporation of in vitro transcribed viral RNA in BHK-21 cells maintained in MEM- $\alpha$ media (Life technologies, Carlsbad, CA) supplemented with 10\% FBS (Life technologies). Freshly harvested viruses mixed with defibrinated sheep blood were presented to 6 to 8-day-old Ae. aegypti RexD WE strain through a Hemotek membrane feeding apparatus (Discovery Workshop, Lancashire, England UK) at $37 \mathrm{C}$ for 60 minutes. Engorged mosquitoes were maintained with $10 \%$ sucrose solution at $16 \mathrm{hr}: 8 \mathrm{hr}$ light-dark photoperiod for 14 days. Mosquitoes were cold anesthetized and dissected to collect the body and secondary tissues including the head, wings and legs of individual mosquitoes at 7,10 , and 14 days post infection (d.p.i.). The secondary tissues were used as the indicator for viral dissemination based on the disseminating phenotypes of the Asibi strain which causes the presence of infectious viruses in the head, wings and legs after the establishment of infection in the midgut [13]. Viral replication kinetics were assessed by titration of homogenized whole mosquitoes by $\mathrm{TCID}_{50}$ as described previously [10]. Infection was defined as the positive immunofluorescence staining in the serially-diluted homogenates of whole mosquito, body or secondary tissues in Vero cells. Dissemination was defined by the positive immunofluorescence staining in the homogenates derived from the secondary tissues in Vero cells. Fisher's exact test was used to analyze the infection and dissemination rates and ANOVA was performed for the analysis of the titers of infected whole mosquitoes.

The infection and dissemination rates for all groups are summarized in Tables 1 and 2, respectively. The T380R mutation did not abolish viral infectivity, as similar infection rates of Asibi strain and Asibi E T380R mutant were observed at $7(60.6 \%$ vs. 66.7\%), $10(50 \%$ vs.58.3\%) and $14(55.3 \%$ vs. $53.8 \%)$ d.p.i. Interestingly, a significantly higher infection rate was observed for the 17D/Asibi M-E E T380R mutant compared to the wildtype $17 \mathrm{D} /$ Asibi M-E chimera at 7 d.p.i. (91.3\% vs. $41.4 \%$,) and 14 d.p.i. $(87.5 \%$ vs. $53.8 \%)(p<0.05)$ although infection rates were not significantly higher at 10 d.p.i. The establishment of infection also led to similar dissemination rates both between the Asibi strain and Asibi E T380R mutant and between the 17D/Asibi M-E chimera and 17D/Asibi M-E E T380R mutant at 7, 10 and 14 d.p.i. Virus titers in whole mosquitoes were not significantly different for any group (Figure 1).

Our results demonstrated the change in the biochemical properties between Thr and Arg did not reduce the viral infectivity and subsequent dissemination of YFV in

Table 2 Ae. aegypti dissemination rates calculated as positive secondary tissues/tested

\begin{tabular}{lllllll}
\hline & \multicolumn{2}{l}{ 7 d.p.i. } & \multicolumn{2}{l}{ 10 d.p.i. } & \multicolumn{2}{l}{ 14 d.p.i. } \\
\hline 17D & $0 / 3$ & $(0.0 \%)$ & $1 / 5$ & $(20.0 \%)$ & $0 / 12$ & $(0.0 \%)$ \\
17D/Asibi M-E & $2 / 6$ & $(33.3 \%)$ & $3 / 8$ & $(37.5 \%)$ & $7 / 14$ & $(50.0 \%)$ \\
17D/Asibi M-E E T380R & $3 / 14$ & $(21.4 \%)$ & $1 / 6$ & $(16.7 \%)$ & $5 / 8$ & $(62.5 \%)$ \\
Asibi & $8 / 17$ & $(47.1 \%)$ & $4 / 5$ & $(80.0 \%)$ & $9 / 15$ & $(60.0 \%)$ \\
Asibi E T380R & $15 / 19$ & $(79.0 \%)$ & $9 / 14$ & $(64.3 \%)$ & $10 / 15$ & $(66.7 \%)$ \\
\hline
\end{tabular}




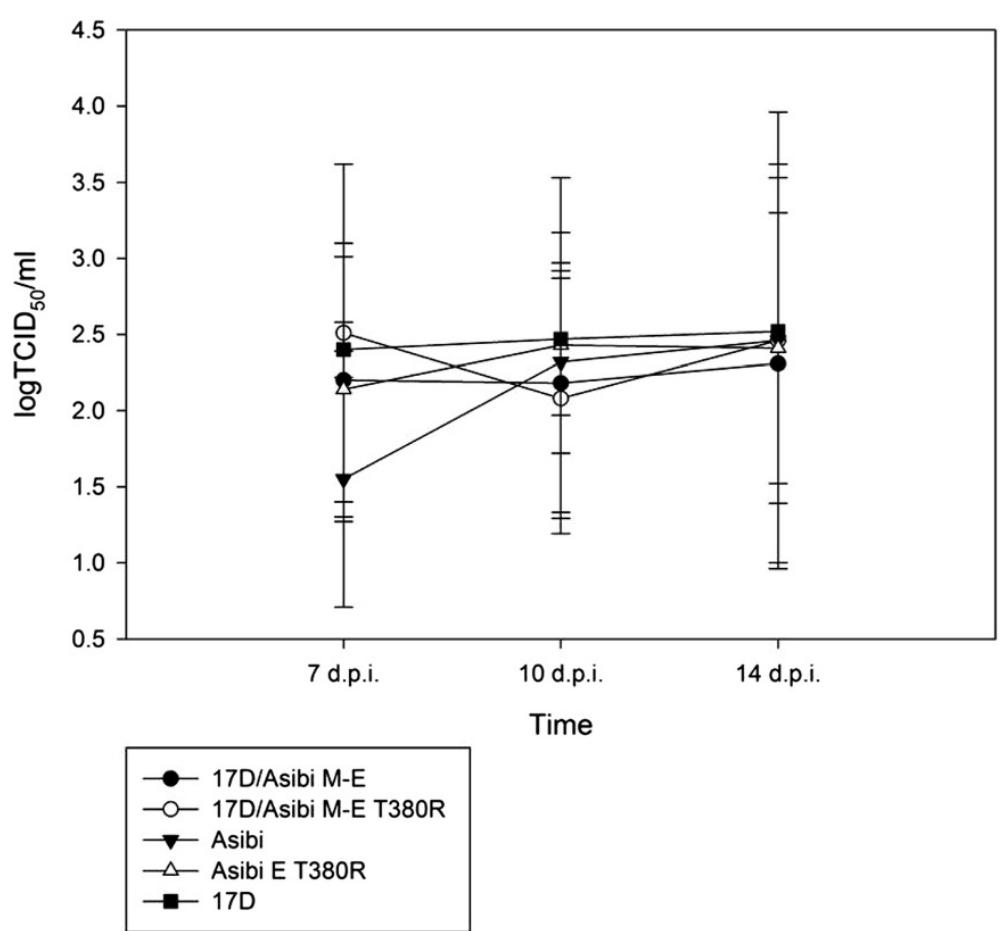

Figure 1 Titers of whole-mosquito homogenates of Ae. aegypti at 7, 10 and 14 d.p.i.

Ae. aegypti. 17D/Asibi M-E E T380R mutant was able to infect a significantly higher percentage of mosquitoes at 7 and 14 d.p.i. than the 17D/Asibi M-E chimera, which was previously shown to have the lower infectivity due to the attenuation by the chimerization [12]. However, the results showed the likelihood of transmission should not be enhanced due to the similar dissemination rates between the wild-type and mutant viruses. The observation was partially consistent with the previously published study on DENV-2 based on the fact that the infectivity was maintained whilst the length of FG-loop was not altered. The more plausible explanation for the increased infectivity due to the T380R mutation may be that the gain of positive charge leads to the more efficient viral attachment to the target cells in the host and the higher infection rates. Although the increase of positive charges on viral structural proteins has been known to reduce the viral dissemination, most mutant viruses still retain the infectivity for susceptible host cells. The presence of the positive charges on the E2 glycoprotein of the mosquito-borne alphavirus eastern equine encephalitis virus led to the higher number of infected brain cells in mice intracerebrally challenged, indicating the presence of positively charged amino acids may be advantageous for the establishment of infection [14]. In the case of adeno-associated virus, the gain of positive charge through the similar $\mathrm{Thr} \rightarrow$ Arg mutation in the capsid region significantly increased the viral attachment to HeLa cells [15]. Our results suggest that the presence of the positively charged arginine on the FG loop may favor the establishment of infection in mosquitoes. Understanding the function of flavivirus RGD motifs and FG loops in viral infection and dissemination can only be achieved by further testing the hypothesis with the reverse genetics systems of Culex-borne flaviviruses, tick-borne flaviviruses and the newly discovered mosquito-only flaviviruses.

\section{Competing interests}

The authors declare that they have no competing interests.

\section{Authors' contributions}

YSH constructed the viral mutants, maintained and infected mosquitoes, performed the virus titrations, conducted statistical analyses, and drafted the manuscript. JTN performed the mosquito infections. KMH participated in data analysis and interpretation and helped to draft the manuscript. ML conceived the study. DV and SH participated in study conception, design and coordination, and directed the research. All authors read and approved the final manuscript.

\section{Acknowledgements}

The authors thank Ms. Jing Huang's expertise in rearing mosquitoes for the use of the experiments.

\section{Author details}

${ }^{1}$ Biosecurity Research Institute, Kansas State University, Manhattan, KS 66506, USA. ${ }^{2}$ Department of Diagnostic Medicine and Pathobiology, College of Veterinary Medicine, Kansas State University, Manhattan, KS 66506, USA.

${ }^{3}$ Medical Countermeasure Systems, Joint Vaccine Acquisition Program, Fort Detrick, MD 21702, USA. ${ }^{4}$ Australian Infectious Disease Research Centre, The University of Queensland, St Lucia, QLD, Australia. 


\section{References}

1. Chen Y, Maguire T, Hileman RE, Fromm JR, Esko JD, Linhardt RJ, Marks RM: Dengue virus infectivity depends on envelope protein binding to target cell heparan sulfate. Nat Med 1997, 3:866-871.

2. Chen Y, Maguire T, Marks RM: Demonstration of binding of dengue virus envelope protein to target cells. J Virol 1996, 70:8765-8772.

3. Hung J-J, Hsieh M-T, Young M-J, Kao C-L, King C-C, Chang W: An external loop region of domain III of dengue virus type 2 envelope protein is involved in serotype-specific binding to mosquito but not mammalian cells. J Virol 2004, 78:378-388.

4. Bhardwaj S, Holbrook M, Shope RE, Barrett AD, Watowich SJ: Biophysical characterization and vector-specific antagonist activity of domain III of the tick-borne flavivirus envelope protein. J Virol 2001, 75:4002-4007.

5. Volk DE, May FJ, Gandham SHA, Anderson A, Von Lindern JJ, Beasley DWC, Barrett ADT, Gorenstein DG: Structure of yellow fever virus envelope protein domain III. Virology 2009, 394:12-18.

6. Lee $E$, Lobigs M: E protein domain III determinants of yellow fever virus $17 \mathrm{D}$ vaccine strain enhance binding to glycosaminoglycans, impede virus spread, and attenuate virulence. J Virol 2008, 82:6024-6033.

7. Lee E, Lobigs M: Mechanism of virulence attenuation of glycosaminoglycan-binding variants of Japanese encephalitis virus and Murray Valley encephalitis virus. J Virol 2002, 76:4901-4911.

8. Lee $\mathrm{E}$, Hall RA, Lobigs M: Common $\mathrm{E}$ protein determinants for attenuation of glycosaminoglycan-binding variants of Japanese encephalitis and West Nile viruses. J Virol 2004, 78:8271-8280.

9. Miller BR, Adkins D: Biological characterization of plaque-size variants of yellow fever virus in mosquitoes and mice. Acta Virol 1988, 32:227-234

10. McElroy KL, Tsetsarkin KA, Vanlandingham DL, Higgs S: Characterization of an infectious clone of the wild-type yellow fever virus Asibi strain that is able to infect and disseminate in mosquitoes. J Gen Virol 2005, 86(Pt 6):1747-1751.

11. Erb SM, Butrapet S, Moss KJ, Luy BE, Childers T, Calvert AE, Silengo SJ, Roehrig JT, Huang CY-H, Blair CD: Domain-III FG loop of the dengue virus type 2 envelope protein is important for infection of mammalian cells and Aedes aegypti mosquitoes. Virology 2010, 406:328-335.

12. McElroy $\mathrm{KL}$, Tsetsarkin KA, Vanlandingham DL, Higgs S: Role of the yellow fever virus structural protein genes in viral dissemination from the Aedes aegypti mosquito midgut. J Gen Virol 2006, 87(Pt 10):2993-3001.

13. McElroy KL, Girard YA, McGee CE, Tsetsarkin KA, Vanlandingham DL, Higgs S: Characterization of the antigen distribution and tissue tropisms of three phenotypically distinct yellow fever virus variants in orally infected Aedes aegypti mosquitoes. Vector Borne Zoonotic Dis Larchmt N 2008, 8:675-687.

14. Gardner $C L$, Ebel GD, Ryman KD, Klimstra WB: Heparan sulfate binding by natural eastern equine encephalitis viruses promotes neurovirulence. Proc Natl Acad Sci USA 2011, 108:16026-16031.

15. Lerch TF, Chapman MS: Identification of the heparin binding site on adeno-associated virus serotype 3B (AAV-3B). Virology 2012, 423:6-13.

doi:10.1186/1743-422X-11-60

Cite this article as: Huang et al:: Mutagenesis analysis of T380R mutation in the envelope protein of yellow fever virus. Virology Journal 2014 11:60

\section{Submit your next manuscript to BioMed Central and take full advantage of:}

- Convenient online submission

- Thorough peer review

- No space constraints or color figure charges

- Immediate publication on acceptance

- Inclusion in PubMed, CAS, Scopus and Google Scholar

- Research which is freely available for redistribution 